\title{
MRI and Ultrasound Fusion Imaging for Cervical Cancer
}

\author{
CLAIRE THÉODORE $^{1}$, JEAN MARC LEVAILLANT $^{1,2}$, PERRINE CAPMAS $^{2}$, NAIMA CHABI $^{3}$, DOUNIA SKALLI ${ }^{1}$, \\ LAURINE VIENET-LEGUÉ ${ }^{1}$, BASSAM HADDAD ${ }^{1}$, HERVÉ FERNANDEZ ${ }^{2}$ and CYRIL TOUBOUL ${ }^{1}$ \\ ${ }^{1}$ Department of Obstetrics and Gynecology, Hôpital Intercommunal de Créteil, \\ Paris Est University, UPEC-Paris XII, Créteil, France; \\ ${ }^{2}$ Department of Obstetrics and Gynecology, Hôpital Bicêtre, Paris Sud University, Paris, France; \\ ${ }^{3}$ Department of Radiology, Centre Hospitalier Intercommunal de Créteil, Créteil, France
}

\begin{abstract}
Background: Evaluating locoregional extension of cervical cancer is a key step in patient management. This study evaluated the feasibility of fusion imaging - a combination of magnetic resonance imaging (MRI) with real-time high-resolution ultrasound (US) - to diagnose cervical cancer and its extension. Patients and Methods: This prospective bi-center study included 13 women who underwent a 1.5-T MRI protocol including at least one T2weighted plane. The results of imaging fusion were then compared with US and MRI results alone. Results: Cervical cancer was detected as a hyperechogenic hypervascularized lesion. Parametrial extension was detected by exploration of the stromal ring and the use of color Doppler mode in fusion imaging, and characterized by visualization of a vascular bridge. Conclusion: Fusion imaging could be used as a complementary technique for MRI to enhance diagnostic performance for cervical cancer lesions. While $M R I$ remains the reference, real-time fusion imaging could improve its characterization and detect parametrial infiltration.
\end{abstract}

Cervical cancer is responsible for more than 266,000 deaths annually and 528,000 new cases were reported worldwide in $2012(1,2)$. The most frequent form of this cancer is squamous-cell carcinoma (SCC), that usually arises consequently to cervical dysplasia linked to human papillomavirus (HPV) infection. Cervical cancer spreads

Correspondence to: Cyril Touboul MD, Ph.D., Department of Obstetrics and Gynecology, Hôpital Intercommunal de Créteil, UMR INSERM U965: Angiogenèse et Recherche translationnelle, 40 avenue de Verdun, Université Paris Est, UPEC-Paris XII, 94000 Créteil, France. Tel: +33 145175543, Fax: +33 145175542, e-mail: cyril.touboul@gmail.com

Key Words: Cervical cancer, fusion imaging, ultrasound, parametrial infiltration, pelvic MRI. through the lymphatic vessels and its natural extension is, thus, primarily towards the parametrium and the vagina. Diagnosis of locally advanced cervical cancer, characterized by a tumor size above $4 \mathrm{~cm}$ and parametrial involvement, is crucial for therapeutic decision-making. Management of these locally advanced cervical cancer cases is based on concurrent chemoradiotherapy $(3,4)$.

The role of pre-therapeutic imaging is therefore central to determining the extension of the cancer and to guiding treatment $(5,6)$. Initially, extension is based on clinical examination according to International Federation of Gynecology and Obstetrics (FIGO) staging (7). However, while magnetic resonance imaging (MRI) is currently used to assess locoregional extension of cervical cancer, there is some discrepancy among clinicians in interpreting the recordings $(8,9)$. This inaccuracy or doubt in interpretation may have substantial impact on patient management.

Over the past 10 years, many studies have evaluated the performances of ultrasonography in the diagnosis of cervical cancer and assessment of extension (10-13). These studies show that ultrasonography is useful and can be as accurate as MRI in this setting (14). However, MRI has the advantage of being easy to review during multidisciplinary tumor board meetings, whereas ultrasonography is dependent on the operator's skill.

In this context, fusion imaging, an emerging technique currently under development, enables real-time visualization of both MRI volumes and high-resolution ultrasonography images simultaneously, as described by Salomon et al. (15). Following promising results in detecting difficult tumors in senology (16), urology and hepatology (17-19), Salomon et $a l$. have shown that fusion imaging is feasible in obstetrics. To date, only limited data are available on fusion imaging in pelvic gynecological oncology.

The objective of this study was to report the feasibility of the use of fusion imaging combining MRI and ultrasonography in diagnosing cervical cancer and assessing extension. 


\section{Patients and Methods}

This prospective bi-center study was conducted between November 2013 and July 2014 at two university Departments of Obstetrics and Gynecology in France: the Hospital Center of Créteil and the Bicetre Hospital. The French Ethics Committee (CPP SUD Mediterranee I) approved the protocol and the cohort of patients with gynecologic al cancer (no. 2016-A01 381-42). We included all women referred for suspicion of cervical cancer. Initial imaging included systematic pelvic MRI (1.5-T) and pelvic ultrasonography. Women in relapse, previously treated by conization or any other surgery, and patients undergoing a different MRI protocol were excluded from the study. We were also unable to include any woman whose surgery had been planned before fusion imaging could be performed.

All the women underwent an initial clinical evaluation during an onco-gynecological consultation. A biopsy of the tumor was taken during this initial consultation, or by colposcopy for infraclinical tumors, and analyzed by anatomopathology for histological diagnosis. Sonography and fusion imaging were performed by experienced reference practitioners blinded to the histological results and FIGO staging.

The first procedure was a classic pelvic examination conducted endovaginally with the bladder partly full using a high frequency probe (6-12 MHz) and a Voluson E8 Ultrasound System (General Electric, Paris, France). The probe was inserted under the cervical canal to maximize the perpendicularity of the beam with the area of interest. The bladder was scanned to the anal canal and the round ligament of the uterus to provide axial and sagittal cross-section recordings. 3D Volume acquisitions with and without Doppler color visualization mode were also recorded. Vascularization was classified subjectively using a Doppler color score (from the International Ovarian Tumor Analysis Group): no flow: color score $=1$; minimal: color $s c o r e=2$; moderate: color $s c o r e=3$; or strong: color score $=4(20)$.

The women then underwent a conventional MRI of the pelvic region using $\mathrm{T} 2$ sequences along the three spatial axes, with diffusion sequences as well as a MRI T1 fat saturated sequence along the axial plane. The Digital Imaging and Communications in Medicine volume dataset was then loaded into fusion ultrasonography equipment (HITACHI PREIRUS or ARIETTA instruments) for manual registration of the live ultrasound and fusion imaging examination.

Visual synchronization of the MRI and ultrasound images was performed by a magnetic field sensor mounted on the ultrasonography probe. The sensor was associated with a field position antenna individually calibrated beforehand for each patient. A magnetic resonance (MR) image centered on the tumor was selected for synchronization with the ultrasound image. The ultrasound examination was then repeated using the fusion imaging probe to scan from the bladder to the anal canal in axial and sagittal cross-section. Doppler color mode was applied for images of interest. The MR images were automatically synchronized and followed the movements of the probe in the three axes.

Exploration along sagittal and axial planes in Doppler color mode enabled characterization of the cervical mass, its extensions and tumoral features. Ultrasonography and fusion imaging were conducted by a skilled operator (JML) with more than 15 years of experience in advanced gynecological ultrasound techniques. The
Table I. Clinicopathological characteristics of the study patients, $n=13$.

\begin{tabular}{lc}
\hline Characteristic & Value \\
\hline Median age (years), (interquartile range) & $56(47-77)$ \\
Maximal tumor diameter (mm), & \\
median (interquartile range) & $41.5(33.75-52.5)$ \\
Tumor stage, n (\%) & $0(0)$ \\
IA & $4(31)$ \\
IB1 & $0(0)$ \\
IB2 & $0(0)$ \\
IIA & $3(23)$ \\
IIB & $2(15)$ \\
III & $3(23)$ \\
IV & \\
Histology, $n(\%)$ & $12(92)$ \\
Squamous cell carcinoma & $1(8)$ \\
Nodular hyperplasia of endocervical glands & $7(54)$ \\
Involvement of parametrium, $n(\%)$ & $5(38)$ \\
Involvement of vagina, $n(\%)$ & $2(15)$ \\
Involvement of bladder, $n(\%)$ & \\
\hline
\end{tabular}

resulting sequences were then analyzed twice, by a seminal reference ultrasonographist and by a radiologist of the pelvic imaging department blinded to the histologic results.

Sociodemographic characteristics and clinicopathological data were recorded for each woman. The diagnosis was established according to anatomopathological results. The diagnosis and disease extension of reference was validated according to the 2009 FIGO classification (7) during a multidisciplinary tumor board meeting. The final diagnosis and extension was compared with imaging results, i.e. classic ultrasonography, MRI and fusion imaging, independently. The criteria studied were: cancer diagnosis, tumor size, and locoregional extension, i.e. parametrial, vaginal and bladder infiltration. We calculated the rate of concordance between the different imaging techniques using Cohen's kappa. Sensitivity, specificity, and positive and negative predictive values of the fusion imaging were calculated.

\section{Results}

Thirteen women were included in the study between November 2013 and July 2014. Twelve of them had a cervical cancer confirmed and documented by histological analysis. Four patients had stage FIGO IB1, three stage IIB, two stage III, one stage IVA and two stage IVB (Table I). The last patient was diagnosed with a nodular hyperplasia of the endocervical glands. The median (interquartile range) age of the 13 women was 56 (47-77) years (Table I).

We found classic ultrasonographic features of cervical cancer in nine of the women (69\%): a hyperechogenic mass, ovoid-shaped, with irregular boundaries and presenting hypervascularization (Figure 1). Three patients (23\%) displayed an isoechogenic tumor (Figure 2). Tumor extension towards the parametrium, usually identified on 


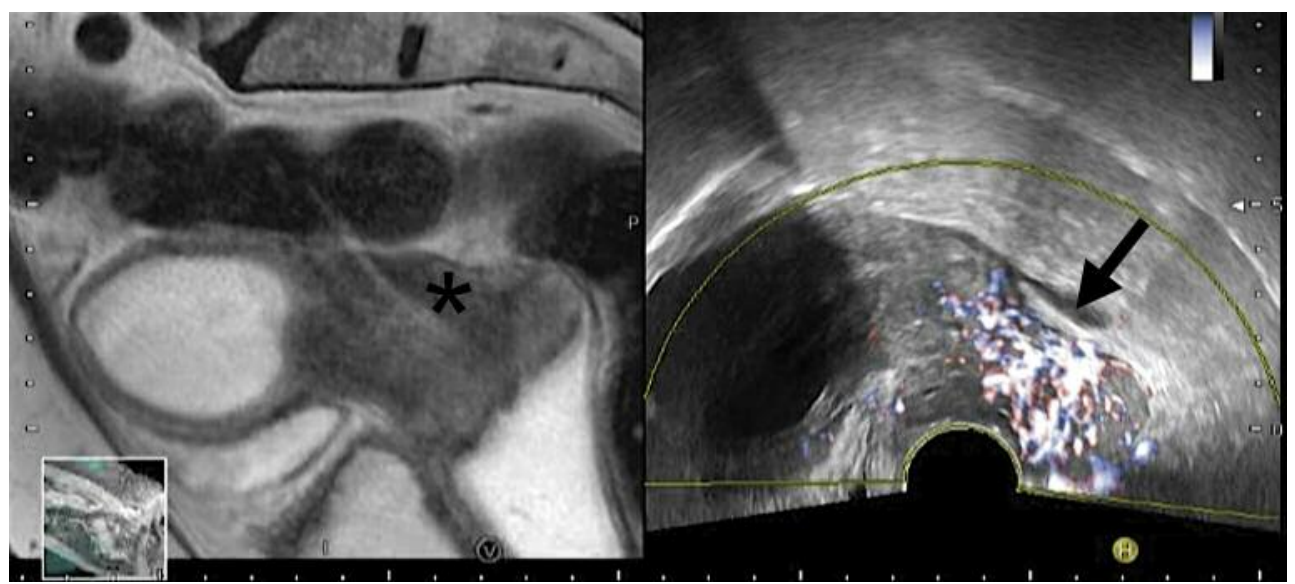

Figure 1. Cervical cancer in fusion imaging, limited to the cervix in the sagittal plane. Left panel: T2-weighted magnetic resonance imaging sequence showing a hyperintense mass ovoid shape with irregular boundaries (*). Right panel: A hyperechogenic hypervascularized mass (arrow) can be seen on ultrasound using color Doppler mode.

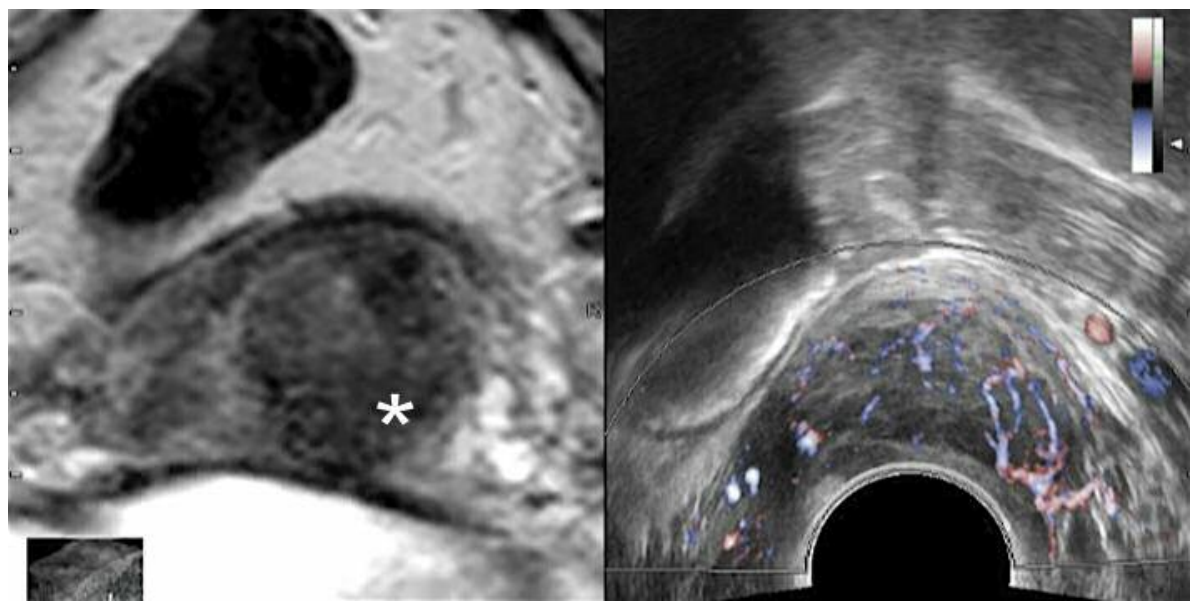

Figure 2. Cervical cancer limited to the cervix in fusion imaging, in axial plane. Left panel: T2-weighted magnetic resonance imaging sequence showing a hyperintense mass $(*)$ without disruption of the stromal ring. Right panel: The mass can be seen to be isoechogenic and hypervascularized on ultrasound.

cervical-centered axial cross-section MRI scans by a disruption of the stromal ring, was also clearly visualized by fusion imaging (Figure 3). The most specific marker of parametrial involvement identified by fusion imaging was the observation of a vascular bridge between the tumor and the parametrium, using color Doppler imaging mode, as depicted in Figure 3.

Vaginal involvement was characterized by a thickening or filling of the vaginal cul-de-sacs (Figure 4). Bladder involvement was defined as an extensive mass infiltrating the bladder associated with a thickening of the bladder wall and the bladder-uterus cul-de-sac with abnormal vascularization. In some women, tumors were also detected in the bladder lumen. These masses had abnormal vascularization when the tumor had extended into the bladder (Figure 5).

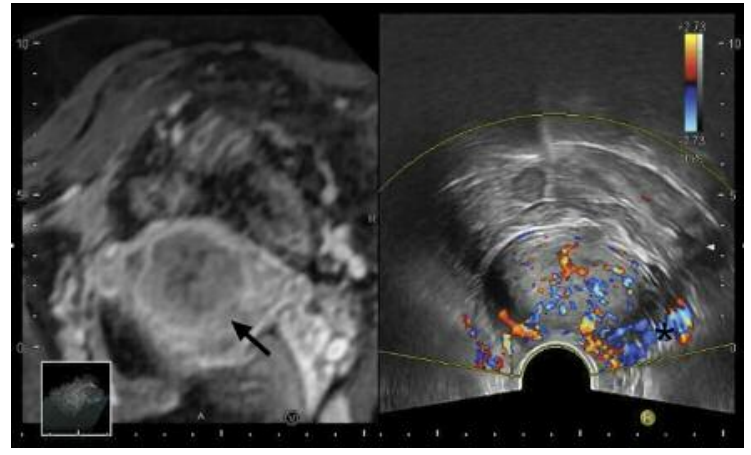

Figure 5. Bladder involvement in fusion imaging, in sagittal plane. Left panel: T2-weighted magnetic resonance imaging sequence with visualization of adenoids in the bladder lumen (arrow). Right panel: On ultrasound, the adenoids were shown to be hypervascularized, using color Doppler mode. 


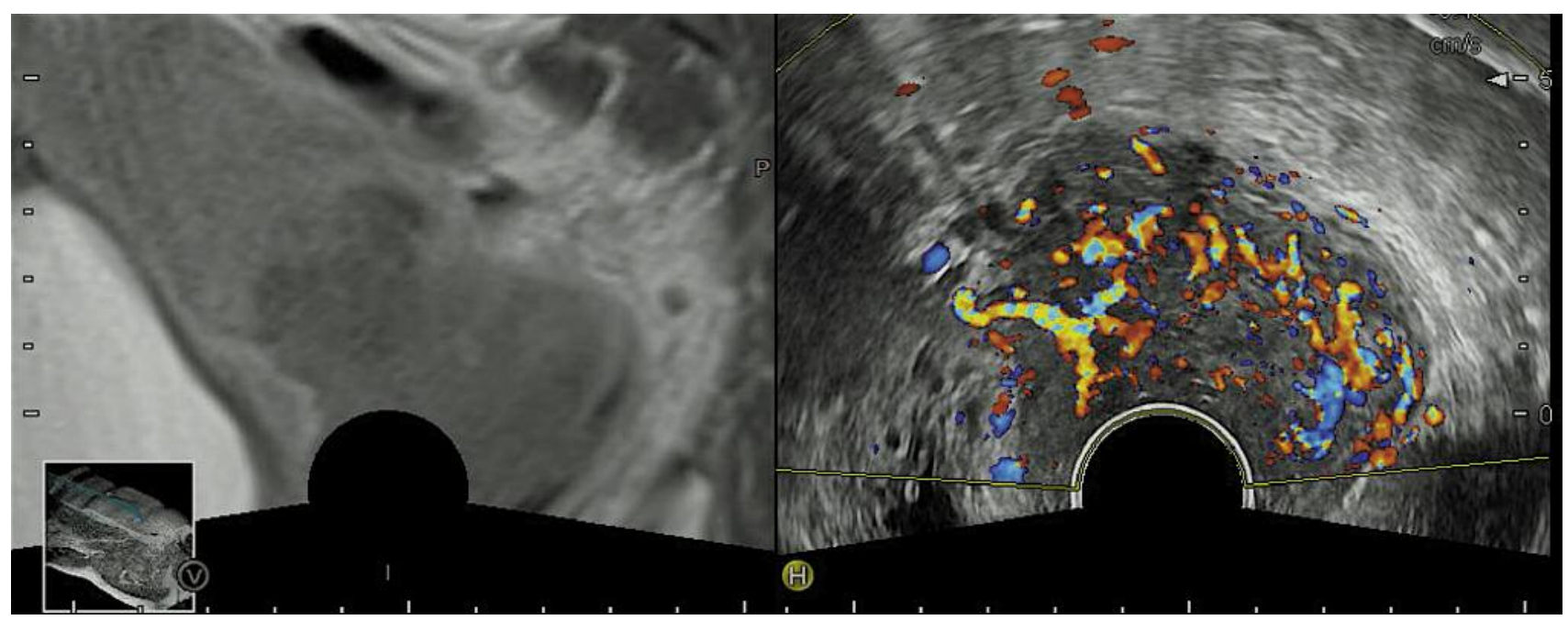

Figure 3. Parametrial infiltration in fusion imaging, in axial plane. Left panel: A T2-weighted magnetic resonance imaging sequence showing the disruption of the cervical stromal ring (arrow). Right panel: On ultrasound, visualization of the vascular bridge between the cervix and the parametrium was achieved by using color Doppler mode.

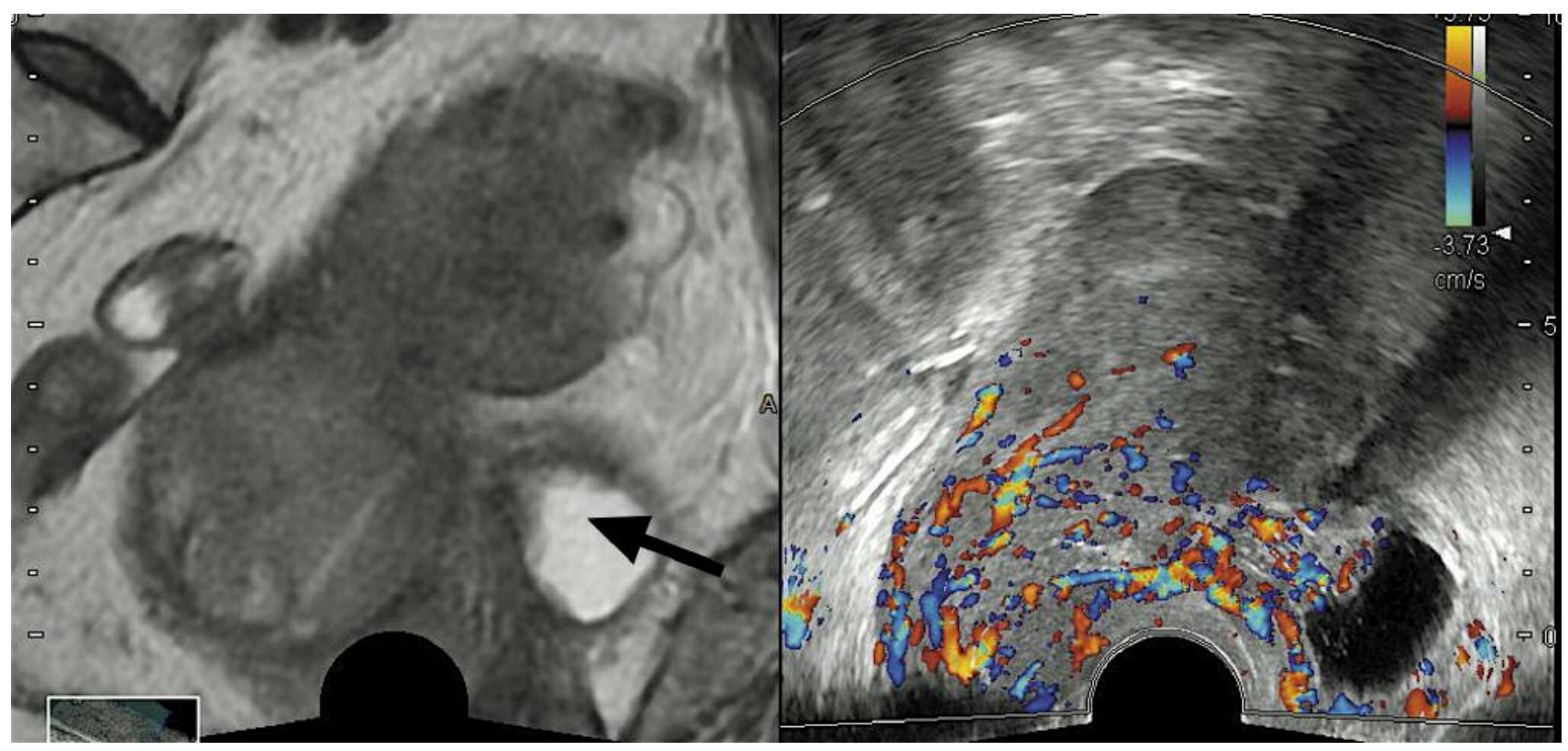

Figure 4. Vaginal involvement in fusion imaging, in sagittal plane. Left panel: T2-weighted magnetic resonance imaging sequence showing a thickening or filling of the vaginal cul-de-sacs. Right panel: On ultrasound, the mass extended to the vagina with abnormal vascularization detected using color Doppler mode.

Fusion imaging examination was successfully completed for all patients within a median (interquartile range) time of 19 (12-29) days following the MRI. Final diagnosis was obtained in all patients (100\%) on fusion imaging and in 12 (92\%) with MRI. The concordance rate between fusion imaging and MRI was $92 \%$.
The median (interquartile range) difference in tumor diameter between MRI and fusion imaging findings was 1.5 (-6-5) $\mathrm{mm}$. Seven patients $(54 \%)$ had parametrial involvement. The concordance rate between classic ultrasonography and fusion imaging was $50 \%$ for parametrial involvement and $85 \%$ between MRI and fusion imaging. 
Table II. Agreement rates between MRI and fusion imaging, $n=13$.

Final diagnosis concordance

\begin{tabular}{lccc}
\cline { 2 - 4 } Criteria & MRI & Fusion imaging & Imaging concordance \\
\hline Malignant tumor diagnosis, n (\%) & 92 & 100 & 92 \\
Cohen's K & 0.628 & 1 & 0.628 \\
Parametrial involvement, n (\%) & 92 & 92 & 85 \\
Cohen's K & 0.843 & 0.847 & 0.697 \\
Vaginal involvement, n (\%) & 77 & 77 & 69 \\
Cohen's K & 0.541 & 0.541 & 0.381 \\
Bladder involvement, n (\%) & 100 & 100 & 100 \\
Cohen's K & 1 & 1 & 1 \\
\hline
\end{tabular}

Both MRI and fusion imaging were concordant with the final diagnosis in $92 \%$ of cases. Involvement of the vagina was observed in $54 \%$ of the patients and was successfully diagnosed in $77 \%$ of cases with both fusion imaging and MRI. The concordance rate was $88 \%$ between classic ultrasonography and fusion imaging and $69 \%$ between MRI and fusion imaging. The bladder was found to be involved in two women $(15 \%)$ (Table II), both of which were diagnosed by classic ultrasonography, MRI and fusion imaging.

\section{Discussion}

This study shows the feasibility and the potential of fusion imaging in patients with cervical cancer. Positive diagnosis was concordant with the histological diagnosis in $100 \%$ of cases using fusion imaging. Fusion imaging successfully identified the malignant cervical masses of a nodular hyperplasia of endocervical glands, which is one of the main differential diagnoses. It was also possible to investigate extension to the parametrium, vagina and the bladder by fusion imaging, which proved helpful in better understanding and interpreting the MRI.

MRI is commonly used to evaluate the extension of gynecological pelvic tumors and our study suggests that fusion imaging could be a complementary tool in the diagnosis and assessment of cervical cancer extension. While fusion imaging does not produce extra images that are not already observable on MRI or classic ultrasonography, it unlocks the potential advantages of combining real-time analysis data from two different techniques. It also opens up the use of MRI as a dynamic examination technique and reduces its artifacts. The synchronous analysis of ultrasound and MRI of the zones of clinical interest, such as the parametrium, may help interpret the MRI images. In the Doppler mode, tumor vascularization was effective in measuring tumor diameter and its borders. Neo-angiogenesis was visualized as intense, heterogeneous and randomly scattered areas of vascularization. By combining MRI and ultrasound in fusion imaging, we also demonstrated successful ultrasound visualization of a disruption in the stromal ring, which is a specific sign of parametrial involvement, usually characterized on MRI by a drastic decrease of the T2 hypo-signal of the fibrous stromal ring (9). This observation was then confirmed by re-interpreting vascular plane images.

While our study highlights the potential of fusion imaging in onco-gynecology, some limitations deserve to be reported. Firstly, the data were obtained in a small population of women with heterogeneous clinical characteristics and disease stage. However, this was a preliminary study aimed at proving feasibility rather than the performance of fusion imaging. Secondly, the patients diagnosed with advancedstage disease did not undergo surgery, in accordance with the established therapeutic care plan. Consequently, the visualized images could not be compared with the histological diagnosis. In this context, the diagnosis of tumor extension was based on the classic clinical examination consisting of palpation of the parametrium. Finally, the fact that fusion imaging requires an operator with expertise in both MRI and ultrasonography restricts its practical application as this skill combination is rarely encountered.

The results presented within the framework of this study are in good agreement with the data found in the literature. The superiority of fusion imaging over MRI alone to diagnose breast cancer and determine local tumor extension has been demonstrated by Nakano et al. (16). These findings were then reproduced by Chang et al. (21) in 2012, after which the use of fusion imaging was extended to other cancer of other organs, especially digestive (17-19, 22), kidney (23) and prostate cancer $(24,25)$. Fusion imaging is also currently being used to guide invasive procedures such as liver biopsy (18) in order to limit the risk of vascular wounds. 
Recent technical developments of ultrasonography have led to its increased use in cervical cancer. Ultrasound diagnosis of cervical cancer has been shown to improve initial diagnosis and evaluation with a better size estimation for small tumors (26, 27). Bellitsos et al. (26) studied vascularization by Doppler and was able to differentiate precancerous lesions from cervical cancer, thus enhancing the diagnostic performance. Our study is concordant with this in showing the use of the Doppler resulted in reinterpretation of the MRI data in the patient with nodular cervical hyperplasia. Fischerova et al. showed that ultrasound was significantly superior to MRI in estimating tumor size with histologic concordant rates of $93.7 \%$ versus $83.2 \%(p<0.006)(27)$. Furthermore, Chiappa et al. compared the use of 3D ultrasound with MRI to assess parametrial infiltration. The percentage concordance between the two techniques was $79 \%(\mathrm{~K}=0.508)$ which confirmed the utility of ultrasound (11). We also improved visualization of parametrial involvement using Doppler by detecting a vascular bridge between the cervical carcinoma and its parametrial extension. This accuracy in diagnosing parametrial involvement has already been reported by a multicenter European trial conducted by Epstein et al. (14). The investigators showed that ultrasound was significantly better than MRI in assessing parametrial invasion in women with early-stage cervical cancer $(\mathrm{K}=0.75$ versus $\mathrm{K}=0.45)$, and also in detecting residual tumors $(\mathrm{K}=0.84$ versus $\mathrm{K}=0.52)(14)$.

In this study, we report the feasibility of using fusion imaging in the diagnosis of cervical cancer and its potential use in the assessment of tumoral extension. Nonetheless, further clinical studies assessing the performance of pelvic fusion imaging are necessary to support the development of this technique in onco-gynecology.

\section{Conflicts of Interest}

Dr C. Touboul declares that he has occasionally acted as a consultant for TWA-ADELPHI.

\section{References}

1 Organization WH: Comprehensive Cervical Cancer Control: A Guide to Essential Practice. Geneva, Switzerland, 2014.

2 Ferlay J, Soerjomataram I, Dikshit R, Eser S, Mathers C, Rebelo M, Parkin DM, Forman D and Bray F: Cancer incidence and mortality worldwide: sources, methods and major patterns in GLOBOCAN 2012. Int J Cancer 136: E359-386, 2015.

3 Green JA, Kirwan JM, Tierney JF, Symonds P, Fresco L, Collingwood $\mathrm{M}$ and Williams CJ: Survival and recurrence after concomitant chemotherapy and radiotherapy for cancer of the uterine cervix: a systematic review and meta-analysis. Lancet 358: 781-786, 2001.

4 Chemoradiotherapy for Cervical Cancer Meta-Analysis C: Reducing uncertainties about the effects of chemoradiotherapy for cervical cancer: a systematic review and meta-analysis of individual patient data from 18 randomized trials. J Clin Oncol 26: 5802-5812, 2008.
5 Haie-Meder C, Fervers B, Fondrinier E, Haugh M, Lhomme C and Guastalla JP: SOR guidelines for concomitant chemoradiotherapy for patients with uterine cervical cancers: evidence update bulletin 2004. Ann Oncol 16: 1100-1108, 2005.

6 Colombo N, Carinelli S, Colombo A, Marini C, Rollo D, Sessa $\mathrm{C}$ and Group EGW: Cervical cancer: ESMO Clinical Practice Guidelines for diagnosis, treatment and follow-up. Ann Oncol 23(Suppl 7): vii27-32, 2012.

7 Petru E, Luck HJ, Stuart G, Gaffney D, Millan D, Vergote I and Gynecologic Cancer I: Gynecologic Cancer Intergroup (GCIG) proposals for changes of the current FIGO staging system. Eur J Obstet Gynecol Reprod Biol 143: 69-74, 2009.

8 Yamashita Y, Baba T, Baba Y, Nishimura R, Ikeda S, Takahashi $\mathrm{M}$, Ohtake $\mathrm{H}$ and Okamura $\mathrm{H}$ : Dynamic contrast-enhanced MR imaging of uterine cervical cancer: pharmacokinetic analysis with histopathologic correlation and its importance in predicting the outcome of radiation therapy. Radiology 216 : 803-809, 2000.

9 Thomassin-Naggara I, Siles P, Balvay D, Cuenod CA, Carette MF and Bazot M: MR perfusion for pelvic female imaging. Diagn Interv Imaging 94: 1291-1298, 2013.

10 Testa AC, Ludovisi M, Manfredi R, Zannoni G, Gui B, Basso D, Di Legge A, Licameli A, Di Bidino R, Scambia G and Ferrandina G: Transvaginal ultrasonography and magnetic resonance imaging for assessment of presence, size and extent of invasive cervical cancer. Ultrasound Obstet Gynecol 34: 335344, 2009.

11 Chiappa V, Di Legge A, Valentini AL, Gui B, Micco M, Ludovisi M, Giansiracusa C, Testa AC and Valentin L: Agreement of two-dimensional and three-dimensional transvaginal ultrasound with magnetic resonance imaging in assessment of parametrial infiltration in cervical cancer. Ultrasound Obstet Gynecol 45: 459-469, 2015.

12 Ghi T, Giunchi S, Kuleva M, Santini D, Savelli L, Formelli G, Casadio P, Costa S, Meriggiola MC and Pelusi G: Threedimensional transvaginal sonography in local staging of cervical carcinoma: description of a novel technique and preliminary results. Ultrasound Obstet Gynecol 30: 778-782, 2007.

13 Epstein E, Di Legge A, Masback A, Lindqvist PG, Kannisto P and Testa AC: Sonographic characteristics of squamous cell cancer and adenocarcinoma of the uterine cervix. Ultrasound Obstet Gynecol 36: 512-516, 2010.

14 Epstein E, Testa A, Gaurilcikas A, Di Legge A, Ameye L, Atstupenaite V, Valentini AL, Gui B, Wallengren NO, Pudaric S, Cizauskas A, Masback A, Zannoni GF, Kannisto P, Zikan M, Pinkavova I, Burgetova A, Dundr P, Nemejcova K, Cibula D and Fischerova D: Early-stage cervical cancer: tumor delineation by magnetic resonance imaging and ultrasound - a European multicenter trial. Gynecol Oncol 128: 449-453, 2013.

15 Salomon LJ, Bernard JP, Millischer AE, Sonigo P, Brunelle F, Boddaert $\mathrm{N}$ and Ville $\mathrm{Y}$ : MRI and ultrasound fusion imaging for prenatal diagnosis. Am J Obstet Gynecol 209: 148 e141-149, 2013.

16 Nakano S, Kousaka J, Fujii K, Yorozuya K, Yoshida M, Mouri Y, Akizuki M, Tetsuka R, Ando T, Fukutomi T, Oshima Y, Kimura J, Ishiguchi $\mathrm{T}$ and Arai O: Impact of real-time virtual sonography, a coordinated sonography and MRI system that uses an image fusion technique, on the sonographic evaluation of MRI-detected lesions of the breast in second-look sonography. Breast Cancer Res Treat 134: 1179-1188, 2012. 
17 Sandulescu DL, Dumitrescu D, Rogoveanu I and Saftoiu A: Hybrid ultrasound imaging techniques (fusion imaging). World J Gastroenterol 17: 49-52, 2011.

18 Jung EM, Schreyer AG, Schacherer D, Menzel C, Farkas S, Loss M, Feuerbach S, Zorger N and Fellner C: New real-time image fusion technique for characterization of tumor vascularisation and tumor perfusion of liver tumors with contrast-enhanced ultrasound, spiral CT or MRI: first results. Clin Hemorheol Microcirc 43: 57-69, 2009.

19 Rennert J, Georgieva M, Schreyer AG, Jung W, Ross C, Stroszczynski $\mathrm{C}$ and Jung EM: Image fusion of contrast enhanced ultrasound (CEUS) with computed tomography (CT) or magnetic resonance imaging (MRI) using volume navigation for detection, characterization and planning of therapeutic interventions of liver tumors. Clin Hemorheol Microcirc 49: 6781, 2011.

20 Kaijser J, Bourne T, Valentin L, Sayasneh A, Van Holsbeke C, Vergote I, Testa AC, Franchi D, Van Calster B and Timmerman D: Improving strategies for diagnosing ovarian cancer: a summary of the International Ovarian Tumor Analysis (IOTA) studies. Ultrasound Obstet Gynecol 41: 9-20, 2013.

21 Chang JM, Han W, Moon HG, Yi A, Cho N, Koo HR, Park IA, Kim SJ, Noh DY and Moon WK: Evaluation of tumor extent in breast cancer patients using real-time MR navigated ultrasound: preliminary study. Eur J Radiol 81: 3208-3215, 2012.

22 Jung EM, Friedrich C, Hoffstetter P, Dendl LM, Klebl F, Agha A, Wiggermann P, Stroszcynski C and Schreyer AG: Volume navigation with contrast enhanced ultrasound and image fusion for percutaneous interventions: first results. PLoS One 7: e33956, 2012
23 Helck A, D'Anastasi M, Notohamiprodjo M, Thieme S, Sommer W, Reiser M and Clevert DA: Multimodality imaging using ultrasound image fusion in renal lesions. Clin Hemorheol Microcirc 50: 79-89, 2012.

24 Wysock JS, Rosenkrantz AB, Huang WC, Stifelman MD, Lepor H, Deng FM, Melamed J and Taneja SS: A prospective, blinded comparison of magnetic resonance (MR) imaging-ultrasound fusion and visual estimation in the performance of MR-targeted prostate biopsy: the PROFUS trial. Eur Urol 66: 343-351, 2014.

25 Fiard G, Hohn N, Descotes JL, Rambeaud JJ, Troccaz J and Long JA: Targeted MRI-guided prostate biopsies for the detection of prostate cancer: initial clinical experience with realtime 3-dimensional transrectal ultrasound guidance and magnetic resonance/transrectal ultrasound image fusion. Urology 81 : 1372-1378, 2013.

26 Belitsos P, Papoutsis D, Rodolakis A, Mesogitis S and Antsaklis A: Three-dimensional power Doppler ultrasound for the study of cervical cancer and precancerous lesions. Ultrasound Obstet Gynecol 40: 576-581, 2012.

27 Fischerova D, Cibula D, Stenhova H, Vondrichova H, Calda P, Zikan M, Freitag P, Slama J, Dundr P and Belacek J: Transrectal ultrasound and magnetic resonance imaging in staging of early cervical cancer. Int J Gynecol Cancer 18: 766-772, 2008.

Received June 12, 2017

Revised July 11, 2017

Accepted July 21, 2017 